\title{
Air pollution and in utero programming of poor fetal growth
}

\author{
"Collection of RNA for analysis of noncoding RNA, protein \\ for histone modifications and plasma for exosomes/ \\ extracellular vesicles is the next frontier of understanding \\ how the environment affects gene regulation."
}

First draft submitted: 5 January 2017; Accepted for publication: 12 January 2017; Published online: 17 February 2017

Keywords: AHRR • air pollution $\bullet$ aryl hydrocarbon receptor repressor $\bullet$ cigarette smoke - DNA methylation • fetal growth • fetal programming • intrauterine growth restriction - $\mathrm{PM}_{2.5} \bullet$ tobacco

Poor fetal growth is associated with adverse postnatal health outcomes. In the newborn period, compared with well-grown infants, those small-for-gestational age (typically defined as less than 10th percentile [1]) are at higher risk of mortality and morbidities including difficulties with glucose homeostasis and thermoregulatory control [2]. They are also more likely to require neonatal intensive care [2]. Subsequently, small-for-gestational age infants are predisposed to hypertension and neurodevelopmental delays and disabilities $[2,3]$. The etiology of poor fetal growth is multifactorial. Maternal conditions such as pre-eclampsia, infections such as cytomegalovirus and toxoplasmosis, maternal malnutrition and exposures to environmental pollutants such as lead, can all lead to infants being born smaller than their genetic potential $[1,4,5]$.

Maternal cigarette smoking in pregnancy represents one of the most preventable causes of poor fetal growth [6]. Air pollution, which contains many of the same compounds found in cigarette smoke including fine particulate matter smaller than five microns in diameter $\left(\mathrm{PM}_{2.5}\right)$, has been shown to increase the risk of many of the same conditions caused by smoking including lung cancer and cardiovascular disease [7]. Further, air pollution exposure in pregnancy is associated with lower birth weight for gestational age [8]. How air pollution affects fetal growth is incompletely understood, but new insights into how the fetal epigenome responds to cigarette smoke may provide clues as to how air pollution may affect the developing fetus.

\section{Smoking \& DNA methylation}

DNA methylation results in differences in phenotype in the absence of DNA sequence variation by regulating transcription. Typically, heavily methylated regions of DNA, usually in promoters, are inaccessible to transcription factors and thus gene expression of these regions is suppressed. Several studies in adults have demonstrated that cigarette smoking results in reproducible DNA methylation differences in blood, specifically demethylation in the promoter of $A H R R$, a gene involved in regulating detoxification processes [9]. Using the Illumina 450K Methylation Beadchip array that measures DNA methylation of over $450,000 \mathrm{CpG}$ sites across the genome, these groups report demethylation of one particular CPG site within AHRR, cg05575921, as most commonly and most significantly associated with smoking. This finding is so consistent that DNA methylation of this site has been proposed as a potentially clinically useful biomarker of smoking, particularly as it reflects smoking status (current, former and never smokers), number of cigarettes smoked, years of smoking and - for former smokers - years since quitting [9]. The University of Iowa and its
Epigenomics
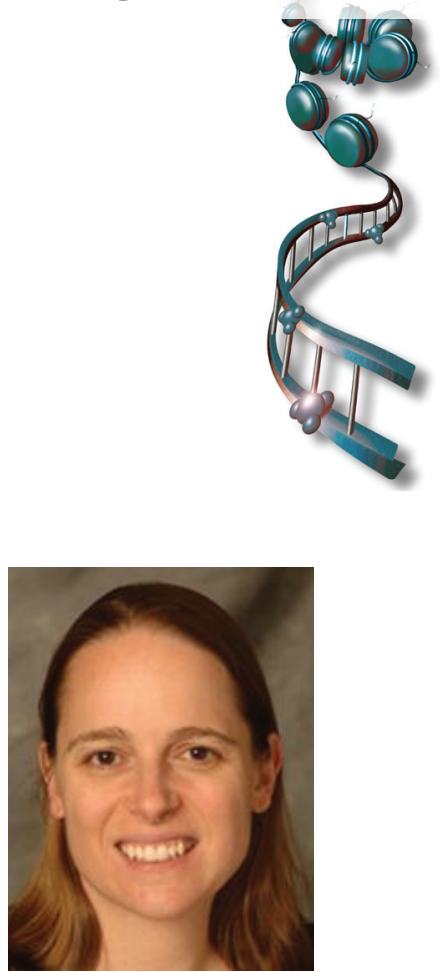

Heather H Burris

Author for correspondence:

Department of Neonatology, Beth Israel Deaconess Medical Center, Boston, MA 02215, USA

Departments of Pediatrics \& Obstetrics, Gynecology \& Reproductive Biology, Harvard Medical School, Boston, MA 02215, USA

Department of Environmental Health, Harvard TH Chan School of Public Health, Boston, MA 02115, USA

Tel.: +1 6176673276

Fax: +1 6176677040

heburris@bidmc.harvard.edu

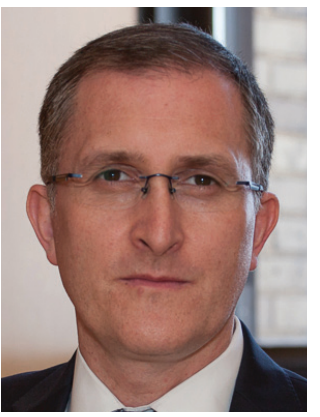

Andrea A Baccarelli Department of Environmental Health Sciences, Mailman School of Public Health, Columbia University, New York, NY 10032, USA

Future $\because \%$ Medicine part of 
investigators have filed intellectual property right claims as well as for a patent of this site as a potentially marketable product to screen for smoking (US Patent $8,637,652)[10]$.

Of particular interest to perinatal researchers is that maternal smoking in pregnancy also affects the DNA methylation of AHRR in offspring cord blood. Joubert and colleagues performed a meta-analysis of 6685 infants from 13 cohorts [11]. The same CpG site in the AHRR gene $(\operatorname{cg} 05575921)$ was the site most significantly associated ( $\mathrm{p}$-value $=1.64 \times 10^{-193}$ ) with maternal smoking. These findings support the hypothesis that maternal exposures may affect fetal development through epigenetic processes including DNA methylation.

\section{Air pollution \& DNA methylation}

Whether less potent but more ubiquitous exposures, such as air pollution, affect DNA methylation or other epigenetic processes in utero is less well established, but plausible. Air pollution, while somewhat distinct from cigarette smoke, shares several of the same components and is associated with many of the same adverse health conditions caused by cigarette smoke [7], including impaired fetal growth [8]. There is also evidence that air pollution in utero can affect infant DNA methylation. Herbstman and colleagues demonstrated in a cohort of 164 mother-infant pairs that maternal personal exposure to polycyclic aromatic hydrocarbons, one marker of diesel exhaust, was associated with infant cord blood global hypomethylation [12]. This finding is consistent with our group's work demonstrating that exposure to black carbon is associated with rapid decrease in DNA methylation in repetitive elements in blood DNA [13].

\section{"Whether less potent but more ubiquitous exposures, such as air pollution, affect DNA methylation or other epigenetic processes in utero is less well established, but plausible."}

Studies of air pollution and fetal growth as well as air pollution and DNA methylation have been primarily observational. These studies may suffer from residual confounding by coexposure to variation in socioeconomic conditions, diet, physical activity and genetics. However, using a double-blind cross-over study of healthy adult volunteers, our group exposed participants for $130 \mathrm{~min}$ to concentrated fine and course particulate matter (PM) and to control medical gas (filtered room air), and then measured blood DNA methylation responses to each of the conditions [14]. There were 2 weeks between exposures, which were randomized with respect to order. After exposure to fine concentrated ambient particles, participants had both significantly lower repetitive element DNA methylation (Alu) and higher systolic blood pressures compared with control gas exposure. These findings confirm that air pollution (at least in the short term) can have effects on DNA methylation and physiologic consequences in adults. The blood pressure finding is particularly relevant when extrapolating to pregnancy because hypertension in pregnancy is associated with decreased fetal growth. Theoretically, the vasoactive properties of $\mathrm{PM}_{2.5}$ might mediate placental insufficiency from air pollution leading to both impaired fetal growth and fetal programming through DNA methylation.

\section{Research gaps \& future of air pollution \& perinatal epigenomics}

One of the most challenging issues faced by air pollution researchers focused on human health is that the doses of PM and gases to which individuals are exposed are much smaller than what is inhaled during active cigarette smoking. Some studies, such as those done by Pope and colleagues, have compared the effects of $\mathrm{PM}_{2.5}$ by various routes (active smoking, passive smoking and air pollution) on specific health outcomes such as cardiovascular mortality, and found that while the doses are substantially lower with air pollution, the increased risk persists despite the route [15]. Researchers at University of California, Berkeley, specifically Muller and colleagues, have compared relative doses and health effects of air pollution to those of active smoking [21]. They report that one cigarette daily is approximately equivalent to a $\mathrm{PM}_{2.5}$ dose of $22 \mu \mathrm{g} / \mathrm{m}^{3}$. Given an average ambient $\mathrm{PM}_{2.5}$ exposure in the USA of $9 \mu \mathrm{g} / \mathrm{m}^{3}$ [22], this would be similar to 0.4 cigarettes daily. In Beijing, where air pollution levels are higher, Muller $e t$ al. propose that exposure can be similar to smoking four cigarettes per day and up to 1.5 cigarettes per hour on a day with particularly high levels of pollution. Therefore, depending on the location of a study, exposures may approach doses of pollutants inhaled during smoking. However, studies of the health implications of air pollution are difficult, not just because of often low levels of exposure in places like the USA, but also because of the ubiquity of exposure among cohorts living within a narrow geographical region. Combining temporal and special variation can improve the detection of associations especially in pregnancy studies where rapid fetal development may be a particularly sensitive time, but large sample sizes will still be required to detect differences in fetal growth and DNA methylation.

Studies detecting differences in mortality, lung cancer and cardiovascular disease with air pollution exposure have typically require sample sizes exceed- 
ing 500,000 [16]. Similarly, studies of birthweight have also required population datasets to detect differences [8]. However, performing methylomic or other types of epigenomic assays, in cohorts that large would be cost prohibitive. Several strategies can be employed to deal with these limitations. First, many cohorts are already analyzing DNA methylation across the genome which can be analyzed with respect to air pollution exposure retrospectively [11]. Our colleagues and others have developed spatiotemporal models to estimate ambient levels of air pollution at the participants' address in many parts of the world [17-19] by partnering with NASA and other governmental agencies to obtain satellite data that can detect $\mathrm{PM}_{2.5}$ and $\mathrm{PM}_{10}$ data. Because these modeling approaches only require information on the participants' location (i.e., participants home address, and if possible other relevant locations or time-activity patterns) to obtain their ambient air pollution levels, collaboration between perinatal researchers and air pollution modeling groups will make larger studies possible, ideally using consortia to perform meta-analyses. Another approach would be to learn from the cigarette smoking literature and create more targeted assays to use a gene-specific approach for regions of interest. Additionally, state laboratories analyze newborn blood for rare, treatable, metabolic conditions using dried blood spots [23]. A group in Southern California has analyzed these dried bloodspots for repetitive element DNA methylation and gene-specific

\section{References}

1 Resnik R. Intrauterine growth restriction. Obstet. Gynecol. 99(3), 490-496 (2002).

2 Pallotto EK, Kilbride HW. Perinatal outcome and later implications of intrauterine growth restriction. Clin. Obstet. Gynecol. 49(2), 257-269 (2006).

3 Gluckman PD, Hanson MA, Cooper C, Thornburg KL. Effect of in utero and early-life conditions on adult health and disease. N. Engl. J. Med. 359(1), 61-73 (2008).

4 Hendrix N, Berghella V. Non-placental causes of intrauterine growth restriction. Semin. Perinatol. 32(3), 161-165 (2008).

5 Rodosthenous RS, Burris HH, Svensson K et al. Prenatal lead exposure and fetal growth: smaller infants have heightened susceptibility. Environ. Int. doi:10.1016/j.envint.2016.11.023 (2016) (Epub ahead of print).

6 Wen SW, Goldenberg RL, Cutter GR et al. Smoking, maternal age, fetal growth, and gestational age at delivery. Am. J. Obstet. Gynecol. 162(1), 53-58 (1990).

7 Brook RD, Rajagopalan S, Pope CA 3rd et al. Particulate matter air pollution and cardiovascular disease: an update to the scientific statement from the American Heart Association. Circulation 121(21), 23312378 (2010). assays, but have not yet reported on sites associated with smoking [20].

\section{"DNA methylation is likely just the tip of the epigenomic iceberg.}

DNA methylation is likely just the tip of the epigenomic iceberg. It is one of the most stable and easily measured of the epigenetic marks, but other mechanisms also regulate gene expression and may be both responsive to air pollution and responsible for poor fetal growth and programming its consequences. Collection of RNA for analysis of noncoding RNA, protein for histone modifications and plasma for exosomes/extracellular vesicles is the next frontier of understanding how the environment affects gene regulation. Ultimately, following cohorts for years to come will allow for understanding the persistence of these marks and their contribution to other long-term health consequences of environmental exposures and fetal growth disturbances.

\section{Financial \& competing interests disclosure}

This work was supported in part by the National Institutes of Health (NIH/NIEHS: K23ES022242, P30ES009089). The authors have no other relevant affiliations or financial involvement with any organization or entity with a financial interest in or financial conflict with the subject matter or materials discussed in the manuscript apart from those disclosed.

No writing assistance was utilized in the production of this manuscript.

Stieb DM, Chen L, Eshoul M, Judek S. Ambient air pollution, birth weight and preterm birth: a systematic review and meta-analysis. Environ. Res. 117, 100-111 (2012).

9 Philibert RA, Beach SR, Brody GH. Demethylation of the aryl hydrocarbon receptor repressor as a biomarker for nascent smokers. Epigenetics 7(11), 1331-1338 (2012).

10 Dogan MV, Shields B, Cutrona C et al. The effect of smoking on DNA methylation of peripheral blood mononuclear cells from African American women. $B M C$ Genomics 15, 151 (2014).

11 Joubert BR, Felix JF, Yousefi P et al. DNA methylation in newborns and maternal smoking in pregnancy: genomewide consortium meta-analysis. Am. J. Hum. Genet. 98(4), 680-696 (2016).

12 Herbstman JB, Tang D, Zhu D et al. Prenatal exposure to polycyclic aromatic hydrocarbons, benzo[a] pyrene-DNA adducts, and genomic DNA methylation in cord blood. Environ. Health Perspect. 120 (5), 733-738 (2012).

13 Baccarelli A, Wright RO, Bollati V et al. Rapid DNA methylation changes after exposure to traffic particles. Am. J. Respir. Crit. Care Med. 179(7), 572-578 (2009).

14 Bellavia A, Urch B, Speck M et al. DNA hypomethylation, ambient particulate matter, and increased blood pressure: 
findings from controlled human exposure experiments. J. Am. Heart Assoc. 2(3), e000212 (2013).

15 Pope CA 3rd, Burnett RT, Krewski D et al. Cardiovascular mortality and exposure to airborne fine particulate matter and cigarette smoke: shape of the exposure-response relationship. Circulation 120(11), 941-948 (2009).

16 Pope CA 3rd, Burnett RT, Thun MJ et al. Lung cancer, cardiopulmonary mortality, and long-term exposure to fine particulate air pollution. JAMA 287(9), 1132-1141 (2002).

17 Just AC, Wright RO, Schwartz J et al. Using high-resolution satellite aerosol optical depth to estimate daily PM2.5 geographical distribution in Mexico city. Environ. Sci. Technol. 49(14), 8576-8584 (2015).

18 Di Q, Kloog I, Koutrakis P, Lyapustin A, Wang Y, Schwartz J. Assessing PM2.5 exposures with high spatiotemporal resolution across the continental United States. Environ. Sci. Technol. 50(9), 4712-4721 (2016).

19 Stafoggia M, Schwartz J, Badaloni C et al. Estimation of daily PM10 concentrations in Italy (2006-2012) using finely resolved satellite data, land use variables and meteorology. Environ. Int. doi:10.1016/j.envint.2016.11.024 (2016) (Epub ahead of print).

20 Breton CV, Yao J, Millstein J et al. Prenatal air pollution exposures, dna methyl transferase genotypes, and associations with newborn line1 and alu methylation and childhood blood pressure and carotid intima-media thickness in the children's health study. Environ. Health Perspect. 124(12), 1905-1912 (2016).

21 Berkeley Earth ${ }^{\mathrm{TM}}$. Air Pollution and Cigarette Equivalence. http://berkeleyearth.org/air-pollution-and-cigaretteequivalence/

22 EPA. EPA's Report on the Environment: Ambient Concentrations of Particulate Matter. https://cfpub.epa.gov/roe/indicator.cfm?i=9\#4

23 CDC. Newborn screening. www.cdc.gov/ncbddd/newbornscreening/ 